\title{
INFLUÊNCIA DA ATIVIDADE ANTIOXIDANTE EM DIFERENTES PERCENTUAIS DE DIESEL/BIODIESEL UTILIZANDO O COMPOSTO ADITIVO OBTIDO A PARTIR DA MORINGA OLEIFERA LAM
}

\author{
L. C. T. de Andrade ${ }^{1}$, F. R. M. França ${ }^{2}$, A. L. D. $\operatorname{Ramos}^{3}$, G. F. da Silva ${ }^{1}$ \\ ${ }^{1}$ Universidade Federal de Sergipe, Departamento de Engenharia Química \\ ${ }^{2}$ Universidade Federal da Bahia, Departamento de Engenharia Química \\ ${ }^{3}$ Universidade Federal de Sergipe, Núcleo de Engenharia Ambiental \\ E-mail para contato: liviacaroline1987@yahoo.com.br
}

\begin{abstract}
RESUMO - Os processos oxidativos no biodiesel ocorrem devido à temperaturas elevadas e a presença de ácidos graxos insaturados. O presente trabalho apresenta parâmetros indicativos da influência antioxidante do composto aditivo da Moringa Oleifera Lam em diferentes percentuais em biodiesel de soja comercial, sob decomposição oxidativa. As amostras foram caracterizadas mediante o estudo oxidativo, através do método Rancimat. Os resultados obtidos mostraram que o tempo de estabilidade oxidativa ultrapassou o limite estabelecido pela ANP (mínimo de 6 horas), chegando a ultrapassar 8 horas de estabilidade.
\end{abstract}

\section{INTRODUÇÃO}

A instabilidade do biodiesel em contato com o ar ambiente (autoxidação), reduzindo sua qualidade durante o armazenamento, tem sido uma preocupação crescente. Nesse sentido, tornase um desafio a produção de biodiesel a partir do óleo de soja, que possui uma baixa estabilidade à oxidação, para manter a qualidade do biodiesel e suas misturas com combustíveis destilados do petróleo durante o longo período de estocagem. A sua determinação em laboratório baseia-se na metodologia de ensaio acelerado originalmente proposta por Hadorn e Zurcher (1974).

A Agência Nacional de Petróleo, Gás e Biocombustíveis (ANP), em sua Resolução nº 14 de 2012, estabelece um limite mínimo de estabilidade à oxidação de 6 horas (ANP, 2013). Atualmente, estudos estão sendo realizados para aplicação de aditivos antioxidantes oriundos de fontes renováveis, adequadas ao cenário ambiental atual e que tenha baixo valor agregado, conforme comenta Thomazini et al. (2011).

A Moringa oleifera Lam é uma planta originária do nordeste indiano que, por ser amplamente utilizada em diversas áreas como na medicina, na agricultura, na alimentação e nas indústrias produtoras de cosméticos, tornou-se natural o seu plantio em diversos países. Além disso, a Moringa possui um óleo de ótima qualidade, que além de servir para a produção de óleo, tem uma excelente estabilidade à oxidação, ultrapassando o limite mínimo estabelecido pela 


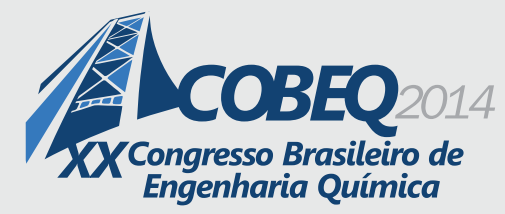

Agência Nacional de Petróleo, Gás e Biocombustíveis (ANP), chegando a atingir 93 horas de estabilidade (PEREIRA, 2011).

Por ser considerado um combustível renovável e de baixo custo, o biodiesel torna-se um produto economicamente competitivo no mercado. Sendo assim, diversos países investem em pesquisas para o desenvolvimento de indústrias produtoras de biodiesel. O Brasil possui uma grande variedade de oleaginosas, e por isso a produção de biodiesel tornou-se uma realidade em um intervalo de tempo bastante curto. Segundo Santos (2008), quando utilizado etanol em sua produção, o biodiesel caracteriza-se como um excelente combustível, pois causa menos danos ao meio ambiente quando comparado à outros combustíveis derivados de petróleo, já que esta substância é considerada menos agressiva ambientalmente, por ser obtida de biomassa.

Os óleos vegetais possuem uma grande variedade de antioxidantes que agem contra a ação de radicais livres responsáveis pela degradação destes, além de bioatividade no organismo humano (CASTELO-BRANCO e TORRES, 2011). Estudos realizados por Pereira et al. (2010) mostraram que o biodiesel produzido através do óleo da Moringa tem uma alta estabilidade e quando se analisou o biodiesel juntamente com extrato coagulante, extraído quimicamente utilizando-se hexano, a estabilidade aumentou consideravelmente.

Segundo Borsato et al. (2012), o biodiesel possui um alto nível de degradação quando em contato com contaminantes inorgânicos ou microbianos, ar atmosférico, altas temperaturas e muita luminosidade. Além destes, há também a preocupação com a sua obtenção a partir de algumas oleaginosas que possuem ácidos graxos em sua composição com um alto grau de insaturação, facilitando a oxidação e dificultando o armazenamento. Para prevenir essa problemática, não ultrapassar o limite máximo de armazenamento nos tanques reduz a quantidade de ar em contato com o combustível.

A estabilidade à oxidação é, portanto, um parâmetro de grande importância para o controle da qualidade do biodiesel e depende de vários fatores e condições, como por exemplo, o manuseio, o processamento e a estocagem.

De acordo com Santos (2008), a estabilidade oxidativa pode ser avaliada através de alguns testes de oxidação acelerada. O método Rancimat é o mais recomendado para análise do biodiesel, podendo-se determinar a estabilidade à oxidação de cada amostra, utilizando-se a temperatura de $110{ }^{\circ} \mathrm{C}$. Neste método ocorre uma decomposição oxidativa ocasionando o envelhecimento prematuro de óleos e gorduras e os produtos formados por esta decomposição são arrastados por um fluxo de ar dentro de uma célula de medição abastecida por água destilada. O tempo de indução é determinado pela medida da condutividade, na qual ocorre um aumento à medida que as reações de oxidação são intensificadas (PEREIRA, 2011).

No entanto, podem ser realizados outros testes para análise oxidativa, tais como a Termogravimetria (TG), Calorimetria Exploratória Diferencial (DSC) e Calorimetria Exploratória Diferencial Pressurizada (PDSC), com o intuito de comparar os parâmetros de oxidação em lubrificantes sintéticos e óleos de aviação, por exemplo (TEIXEIRA, 2010). 


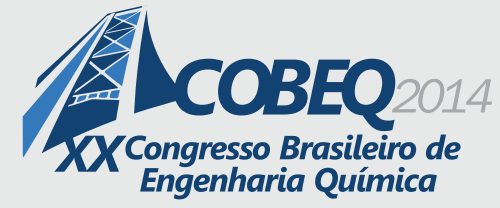

Os óleos e as gorduras podem ser utilizados como fontes de energia e vitaminas importantes para a saúde humana. No entanto, estão propensos a processos oxidativos causadores de efeitos tais como odores e a formação de compostos tóxicos que provocam certas restrições quanto ao seu uso. A partir disso, surge a idéia de se utilizar substâncias antioxidantes, podendo estas serem naturais ou sintéticas, que ajudam a controlar ou minimizar problemas de degradação. (CORDEIRO et al., 2013).

Conforme comentam Thomazini et al. (2011), substâncias antioxidantes também podem ser encontradas em pequenas quantidades nos alimentos, tais como frutas, vegetais, cereais e especiarias. Diversos estudos mostram que os antioxidantes naturais são mais procurados pelas indústrias de alimentos pela sua importância na preservação de óleos e gorduras, no que diz respeito ao controle da ação de radicais livres que causam a degradação destes, além de não ser prejudicial à saúde humana.

Assim como os alimentos o biodiesel está suscetível à oxidação, ao contrário do diesel fóssil, e para garantir a sua estabilidade oxidativa pode-se utilizar, além de aditivos naturais, os aditivos sintéticos, tais como butil hidroxitolueno (BHT), butil-hidroxianisol (BHA), galato de propila (GP) e terc-butil hidroquinona (TBHQ) (CASTELO-BRANCO e TORRES, 2011).

Este trabalho tem como objetivo avaliar a influência do composto antioxidante de baixo custo proveniente da Moringa oleifera Lam, como aditivo natural no biodiesel de soja com baixa estabilidade à oxidação.

\section{METODOLOGIA}

A moringa utilizada é de cultivo próprio da Universidade Federal de Sergipe no Campus "Professor José Aloísio de Campos", situado em São Cristóvão. Desta foram separadas as sementes com a casca, logo após cominuídas em um triturador e em estufa com circulação de ar, com temperatura igual a $40{ }^{\circ} \mathrm{C}$ foi realizado o processo de secagem durante um período de 24 horas. É importante ressaltar que a temperatura não pode ultrapassar este valor para que não haja perda nas propriedades físico-químicas da matéria-prima.

Conforme a metodologia utilizada por Pereira (2010), o processo para obtenção do composto aditivo da moringa para conferir alta estabilidade foi realizado pela rota de transesterificação in situ utilizando-se álcool etílico, com proporção adequada óleo/álcool (1:161) em mistura com um catalisador básico, sendo este o hidróxido de sódio $(\mathrm{NaOH})$, juntamente com o pó das sementes da moringa com casca. Para todas as soluções, foram adicionados $200 \mathrm{~g}$ do pó na mesma temperatura e $6 \%$ do catalisador básico. É importante salientar que a moringa contém um teor de óleo de 35 a $40 \%$ na semente com casca, ou seja, cerca de 70 g de óleo em cada extração.

O processo de mistura da solução foi conduzido em um reator batelada agitado com duração em torno de trinta minutos ou até ocorrer a dissolução do catalisador. Após esta etapa foi adicionado o pó das sementes com casca ao misturador que realizou a homogeneização dos 
materiais reacionais por $2 \mathrm{~h}$ e $30 \mathrm{~min}$ a uma temperatura de $25{ }^{\circ} \mathrm{C}$. Em seguida, a mistura foi separada por filtração a vácuo, na qual foi retirado o filtrado composto aditivo da moringa e a torta, sendo esta utilizada como meio filtrante de água produzida por outro grupo de estudo. Ainda com álcool, o filtrado foi colocado em um rotaevaporador, com temperatura igual a $60{ }^{\circ} \mathrm{C}$, para que este fosse totalmente recuperado e facilitasse a separação.

Então, livre de álcool, o filtrado foi colocado em um funil de decantação por 24 horas, para que ocorresse a separação de biodiesel e glicerina. Para o processo de lavagem foi utilizado água destilada e logo após esta etapa este passou por um processo de centrifugação para eliminar sais e impurezas. Em seguida o biodiesel foi seco em estufa a $100{ }^{\circ} \mathrm{C}$ para eliminação da umidade residual.

Para verificar a eficiência do composto aditivo foi realizado um estudo da estabilidade oxidativa pelo método Rancimat. Uma suspensão foi preparada com $3 \mathrm{~g}$ de cada amostra e $50 \mathrm{~mL}$ de água deionizada, aquecidos a $110^{\circ} \mathrm{C}$. Primeiramente foi feito um teste de estabilidade com o biodiesel de soja puro para avaliar a sua estabilidade sem o aditivo e posteriormente foi avaliada após a adição desse. É importante comentar que a ANP tem interesse em aumentar o tempo de estabilidade para 8 horas e a melhor concentração do composto deve atender a este limite.

\section{RESULTADOS}

A Figura 1 apresenta o perfil da estabilidade à oxidação do biodiesel de soja puro. Pode-se observar a baixa estabilidade deste biodiesel $(5,35 \mathrm{~h})$, indicando ser um material adequado para ser utilizado neste estudo, com base na ANP.

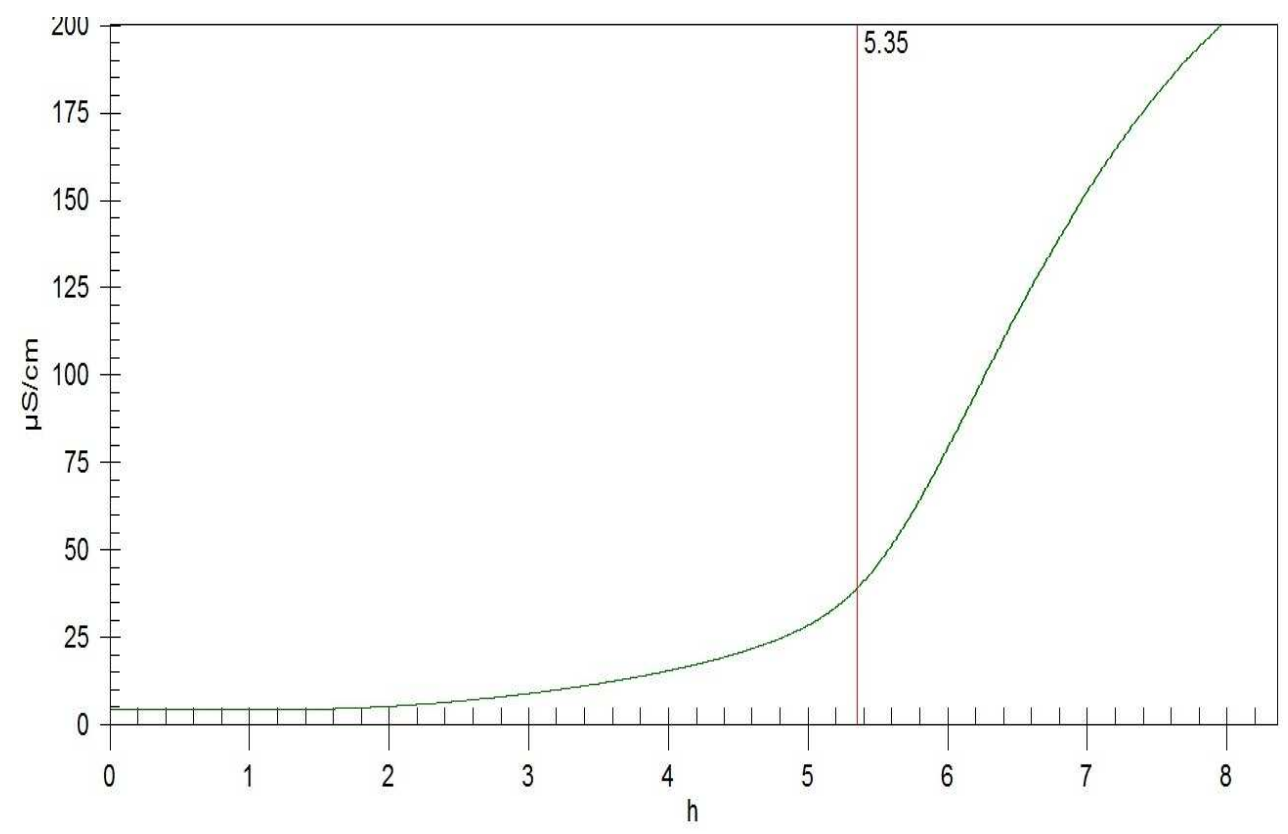

Figura 1 - Estabilidade à Oxidação do biodiesel de soja puro. 
A Figura 2 apresenta o perfil da estabilidade de biodiesel de soja em mistura com o aditivo obtido a partir da moringa com concentração igual a 1000 ppm. Pode-se observar que utilizandose a menor concentração do aditivo, é possível obter um aumento significativo na estabilidade do biodiesel de soja, passando de 5,35 h para $6,85 \mathrm{~h}$.

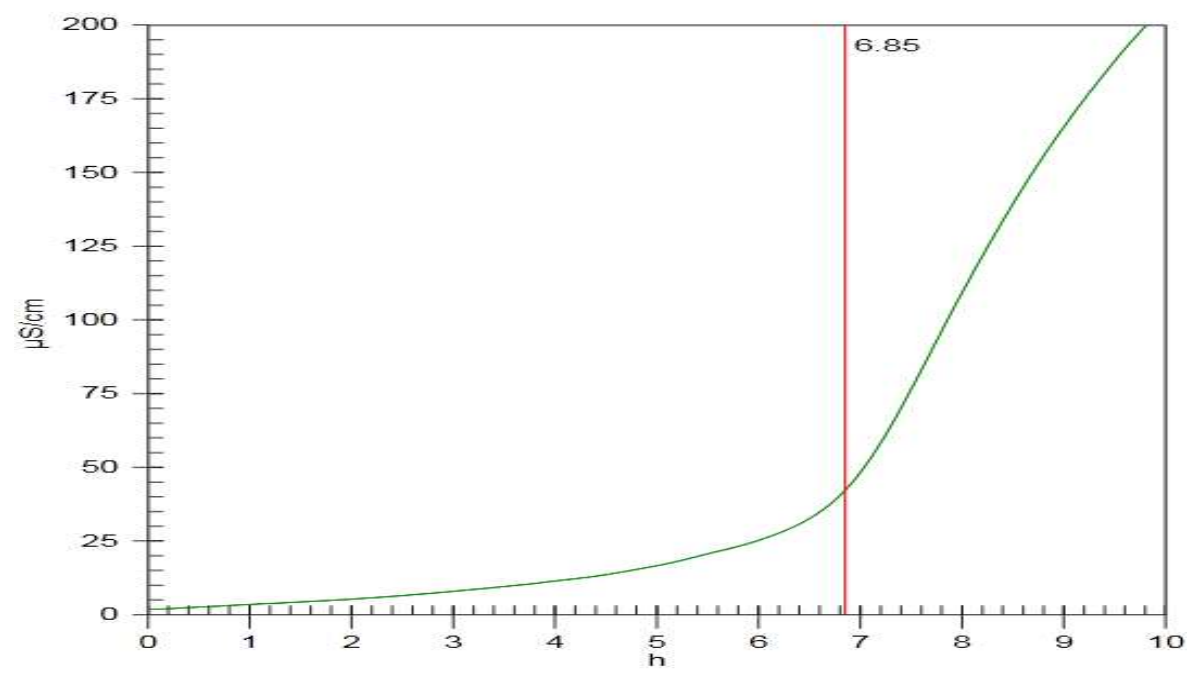

Figura 2 - Estabilidade à Oxidação do biodiesel de soja com 1000 ppm de aditivo.

Para a concentração de aditivo igual a 5000 ppm obteve-se um aumento atingindo o tempo de estabilidade igual a 7,64 h, aproximando-se do tempo estimado pela ANP. Já na terceira concentração, sendo essa de 7500 ppm obteve-se estabilidade durante 8,23 h, chegando a ultrapassar tal estimativa, conforme mostrado nas Figuras 3 e 4, respectivamente.

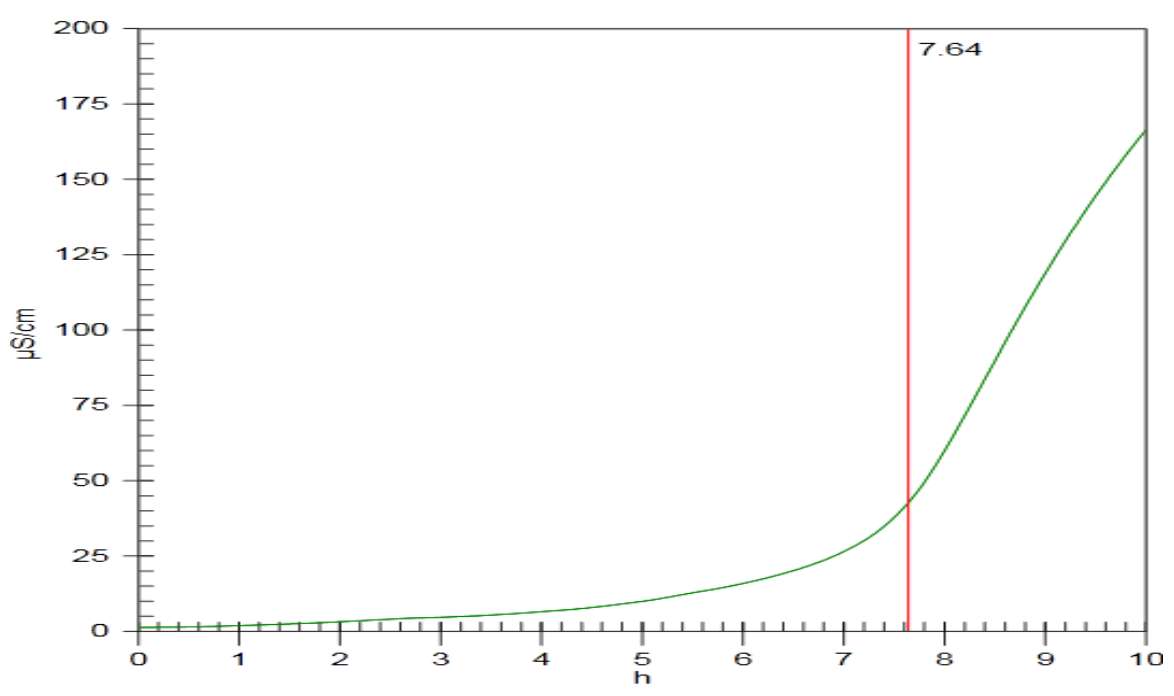

Figura 3 - Estabilidade à Oxidação do biodiesel de soja com 5000 ppm de aditivo. 


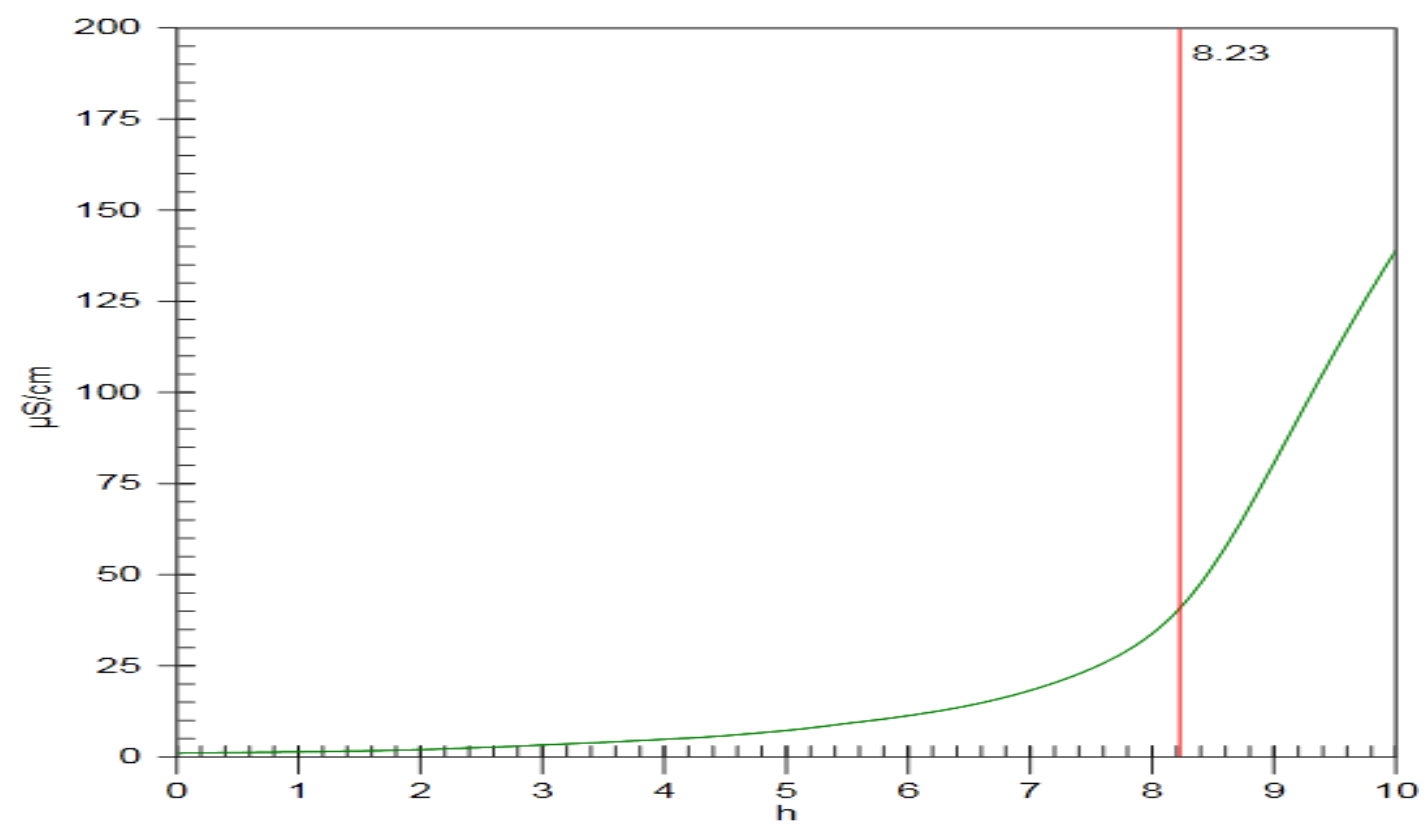

Figura 4 - Estabilidade à Oxidação do biodiesel de soja com 7500 ppm de aditivo.

Analisando-se os resultados, pode-se concluir que quanto maior for a concentração de aditivo, maior será o tempo de estabilidade à oxidação do biodiesel ao qual este for adicionado. $\mathrm{E}$ com base no Decreto 5.448/2005 que autoriza a adição de até $2 \%$ em volume, o biodiesel de moringa mostrou ser um aditivo eficiente, já que com apenas $0,75 \%$ adicionado ao biodiesel de soja, esse atingiu um tempo de estabilidade excelente.

\section{CONCLUSÕES}

O estudo da estabilidade do biodiesel da Moringa foi escolhido por ser de baixo valor agregado, pois este requer menores custos de produção. A reação de transesterificação nesse processo utilizou óleo de moringa, álcool etílico e hidróxido de sódio como regente e variaram-se parâmetros como as concentrações do aditivo. Com a finalização deste trabalho, observou-se que o biodiesel da Moringa oleifera LAM pode ser utilizado como aditivo antioxidante, a fim de aumentar a estabilidade oxidativa de biodieseis oriundos de outras fontes.

\section{REFERÊNCIAS}

AGENCIA NACIONAL DE PETRÓLEO - ANP. RESOLUÇÃO ANP Nº 14, DE 11.05.2012 - DOU 18.05.2012, disponível

http://nxt.anp.gov.br/nxt/gateway.dll/leg/resolucoes_anp/2012/maio/ranp\%2014\%20-\%202012.xml, consultado em 28/08/2013.

BORSATO, D.; MAIA, E. C. R.; DALL'ANTONIA, L. H.; SILVA, H. C. DA; PEREIRA, J. L. Cinética da Oxidação de Biodiesel de Óleo de Soja em Mistura com TBHQ: determinação do tempo de estocagem. Rev. Quí. Nov. vol.35/ no. 4 /São Paulo, 2012. 
CASTELO-BRANCO, V. N.; TORRES, A. G. Capacidade Antioxidante Total de Óleos Vegetais Comestíveis: determinantes químicos e sua relação com a qualidade dos óleos. Rev. de Nut., vol. 24/ n.1/ Campinas jan./fev, 2011.

CORDEIRO, A. M. T. M.; MEDEIROS, M. L.; SILVA, M. A. A. D.; SILVA, I. A. A.; SOLEDADE, L. E. B; SOUZA, A. L.; QUEIROZ, N.; SOUZA, A. G. Rancimat and PDSC Accelerated Techniques for Evaluation of Oxidative Stability of Soybean Oil With Plant Extracts. J. Thermal Analysis Calorimetry, 2013.

HADORN, H.; ZURCHER, K.; Zurbestimmung der Oxydationsstabilitat Von Olen und Fetten. Deustsche Ledensmittel Rundschau, v. 70, n. 2, p. 57-65, 1974.

PEREIRA, D. F.; VASCONCELOS, V. M.; VIEIRA, A. C.; ARANDA, D. A. G.; SILVA, G. F. Estudo da Estabilidade à Oxidação do Biodiesel a Partir da Moringa. II Encontro Nacional de Moringa. Universidade Federal de Sergipe, Aracaju-SE, Brasil, 2010.

PEREIRA, D. F. Potencialidades da Moringa oleifera LAM na Produção de Biodiesel e no Tratamento de Água Produzida na Extração de Petróleo. Dissertação (Mestrado em Engenharia Química) - Programa de Pós-Graduação em Engenharia Química, Universidade Federal de Sergipe, Aracaju-SE, Brasil, 2011.

SANTOS, J. R. J. Biodiesel de Babaçu: Avaliação Térmica, Oxidativa e Misturas Binárias. Tese (Doutorado em Química) - Programa de Pós-Graduação em Química, Universidade Federal da Paraíba, João Pessoa-PB, Brasil, 2008.

TEIXEIRA, G. A. A. Avaliação do Tempo de Vida Útil de Biodiesel Metílico Obtido a partir da Mistura de Sebo Bovino e Óleos de Soja e Babaçu. Tese (Doutorado em Química) - Programa de Pós-Graduação em Química, Universidade Federal da Paraíba, João Pessoa-PB, Brasil, 2010.

THOMAZINI, M. H.; KLAGENBOECH, R.; MOTTA, C. V.; LENZ, G. F.; ZARA, R. F. Antioxidantes Sintéticos e Naturais Aplicados em Óleo Vegetal sob Condições de Oxidação. III Encontro de Divulgação Científica e Tecnológica. Universidade Tecnológica Federal do Paraná, Curitiba-PR, Brasil, 2011. 